\title{
ENERGY TRANSFER OF $O\left({ }^{1} D\right)$ ATOMS IN COLLISION WITH $O\left({ }^{3} \mathrm{P}\right)$ ATOMS
}

\author{
JENG-HWA YEE \\ Space Physics Research Laboratory, University of Michigan, Ann Arbor, MI 48109, U.S.A. \\ and \\ A. Dalgarno \\ Harvard-Smithsonian Center for Astrophysics, 60 Garden Street, Cambridge, MA 02138, U.S.A.
}

(Received 13 May 1986)

\begin{abstract}
Calculations are carried out of the elastic scattering and excitation exchange cross-sections in collisions of $\mathrm{O}\left({ }^{1} \mathrm{D}\right)$ and $\mathrm{O}\left({ }^{3} \mathrm{P}\right)$ atoms, which determine the degree of thermalization of the $O\left({ }^{1} \mathrm{D}\right)$ atoms produced by dissociative recombination in the thermosphere. The effective elastic scattering and excitation exchange cross-sections are calculated to be $1.55 \times 10^{-15}$ and $6.25 \times 10^{-16} \mathrm{~cm}^{2}$, respectively, at a relative collision energy of $1.0 \mathrm{eV}$. The mutual diffusion coefficient between $O\left({ }^{1} \mathrm{D}\right)$ and $O\left({ }^{3} \mathrm{P}\right)$ atoms is also presented.
\end{abstract}

\section{INTRODUCTION}

Metastable $O\left({ }^{1} \mathrm{D}\right)$ atoms are the source of the $6300 \AA$ red line emission in the airglow. In the $F$-region, the $O\left({ }^{\prime} D\right)$ atoms are produced at night by the dissociative recombination of $\mathrm{O}_{2}^{+}$ions through three possible channels :

$$
\begin{aligned}
\mathrm{O}_{2}^{+}\left(X^{2} \Pi_{g}\right)+\mathrm{e} & \rightarrow \mathrm{O}\left({ }^{1} \mathrm{D}\right)+\mathrm{O}\left({ }^{1} \mathrm{~S}\right) \\
& \rightarrow \mathrm{O}\left({ }^{1} \mathrm{D}\right)+\mathrm{O}\left({ }^{1} \mathrm{D}\right) \\
& \rightarrow \mathrm{O}\left({ }^{1} \mathrm{D}\right)+\mathrm{O}\left({ }^{3} \mathrm{P}\right)
\end{aligned}
$$

The reactions are exothermic with excess energies of $0.83,3.1$ and $5.0 \mathrm{eV}$ in channels 1,2 and 3 , respectively, assuming that $\mathrm{O}_{2}^{+}$ions are in their lowest vibrational level. The excess energy appears as the translational energy of the product atoms and is much greater than the ambient thermal energy. The steady state energy distribution of the $O\left({ }^{\prime} D\right)$ atoms thus depends upon the thermalization collision frequency which is determined by the cross-sections for scattering of the $O\left({ }^{1} D\right)$ atoms by ground state $\mathrm{O}\left({ }^{3} \mathrm{P}\right)$ atoms.

The $O\left({ }^{1} \mathrm{D}\right)$ atoms have a radiative lifetime of about 110 s (Garstang 1951; Froese-Fischer and Saha, 1983). It has been assumed that $O\left({ }^{\prime} D\right)$ atoms are completely thermalized in the region of maximum production near $250 \mathrm{~km}$ so that the observed 6300 $\AA$ emission line shape may be used to monitor the thermospheric neutral temperature (Biondi and Feibelman, 1968 ; Thuillier et al., 1977 ; Hernandez, 1980 ; Biondi and Meriwether, 1985).

Non-thermal signatures were reported by Schmitt et al. (1981) and Ignatyev et al. (1984). Schmitt et al. found that at altitudes above $600 \mathrm{~km}$ the emission altitude profile departs from the diffusive equilibrium profile, and they attributed the departure to the incomplete thermalization of $\mathrm{O}\left({ }^{\mathrm{l}} \mathrm{D}\right)$ at high altitudes. Their conclusion was based on model calculations which assumed that the non-thermal $\mathrm{O}\left({ }^{1} \mathrm{D}\right)$ atoms are thermalized in a single collision with ambient ground state $\mathrm{O}\left({ }^{3} \mathrm{P}\right)$ atoms. A thermalization collision crosssection of $1.5 \times 10^{-15} \mathrm{~cm}^{2}$ was derived from the data.

In practice, two types of thermalization collisions occur between an $O\left({ }^{1} \mathrm{D}\right)$ atom and an $O\left({ }^{3} \mathrm{P}\right)$ atom. The two atoms may exchange their identities with no transfer of momentum or energy in an excitation exchange collision, as Schmitt et al. (1981) assumed, and they may be scattered in an elastic collision (Mott and Massey, 1965). Although excitation exchange collisions are very efficient in thermalizing the kinetically hot $O\left({ }^{1} D\right)$ atoms, the cross-sections are not equal to the total collision cross-sections. The gradual thermalization through elastic scattering has also to be considered. In this paper, we calculate the two crosssections and evaluate the efficiency of energy transfer in the collisions of $O\left({ }^{1} \mathrm{D}\right)$ and $O\left({ }^{3} \mathrm{P}\right)$ atoms.

Another consequence of the long radiative lifetime is the importance of transport processes. At high altitudes, the distribution of the $O\left({ }^{1} \mathrm{D}\right)$ atoms is controlled by diffusion. The magnitude of the diffusion cross-sections determines where diffusion becomes dominant. We present calculations of the diffusion cross-sections and the mutual diffusion coefficient for $O\left({ }^{1} \mathrm{D}\right)$ atoms moving in an $O\left({ }^{3} \mathrm{P}\right)$ atmosphere. 
TABle 1. THE INTERACtion POtEntials that SEPaRated to $\mathrm{O}\left({ }^{3} \mathrm{P}\right)+\mathrm{O}\left({ }^{\prime} \mathrm{D}\right)$

\begin{tabular}{ccc}
\hline $\begin{array}{c}\text { Separaled-atom } \\
\text { limit }\end{array}$ & $\begin{array}{c}\text {-Symmetry } \\
\text { states }\end{array}$ & $\begin{array}{c}u \text {-Symmetry } \\
\text { states }\end{array}$ \\
\hline $\mathrm{O}\left({ }^{3} \mathrm{P}\right)+\mathrm{O}\left({ }^{1} \mathrm{D}\right)$ & ${ }^{3} \Sigma_{g}^{+}, 2^{3} \Sigma_{g}^{-}, 3^{3} \Sigma_{g}^{-}$ & ${ }^{3} \Sigma_{u}^{-}, \mathbf{B}^{3} \Sigma_{u}^{-}, 2^{3} \Sigma_{u}^{-}$ \\
& $2^{3} \Pi_{g}, 3^{3} \Pi_{g}, 4^{3} \Pi_{g}$ & $2^{3} \Pi_{u}, 3^{3} \Pi_{u}, 4^{3} \Pi_{u}$ \\
& $1^{3} \Delta_{g}, 2^{3} \Delta_{g}$ & $2^{3} \Delta_{u}, 3^{3} \Delta_{u}$ \\
& ${ }^{3} \Phi_{g}$ & ${ }^{3} \Phi_{u}$ \\
\hline
\end{tabular}

\section{COLLISION CROSS-SECTIONS}

Because of the symmetry, an excited atom and a normal atom of the same kind may interact in either of two distinct ways, depending on whether the electronic wave function for the combined system is symmetrical or anti-symmetrical with respect to the interchange of the nuclei (Mott and Massey, 1965). For the case of $O\left({ }^{1} \mathrm{D}\right)$ and $O\left({ }^{3} \mathrm{P}\right)$ atoms, the atoms may approach along any one of the $18 \mathrm{O}_{2}$ molecular symmetry states listed in Table 1 . The differential crosssection $I(\theta, v)$ for elastic scattering at a relative velocity $v$ may be written as the weighted sum

$$
I(\theta, v)=\sum_{j} g_{j}\left|f_{j}(\theta)\right|^{2}
$$

where $g_{j}$ is the statistical weight of the $j$ th channel, $j$ being the molecular symmetry label, and $f_{j}(\theta)$ is the scattering amplitude at the scattering angle $\theta$ corresponding to the interaction potential $V_{j}$. In terms of the elastic scattering phase shifts $\eta_{i}^{j}$ with angular momentum quantum number $l$,

$$
f_{j}(\theta)=\frac{1}{2 i k} \sum_{l}(2 l+1)\left\{\exp \left(2 i \eta_{l}^{j}\right)-1\right\} P_{l}(\cos \theta)
$$

where $k$ is the wave number. If $\mu$ is the reduced mass, $k=\mu v / h$.

If we denote the interaction energies by $V^{+}(r)$ for the symmetric state and $V^{-}(r)$ for the anti-symmetric state, where $r$ is the internuclear distance, the total collision cross-sections $\sigma$ and the cross-sections for excitation exchange $\sigma_{\mathrm{tr}}$ and for elastic scattering $\sigma_{\mathrm{el}}$ are given by

$$
\begin{gathered}
\sigma=\left(2 \pi / k^{2}\right) \sum_{l}(2 l+1)\left(\sin ^{2} \eta_{l}^{+}+\sin ^{2} \eta_{l}^{-}\right) \\
\sigma_{\mathrm{tr}}=\left(\pi / k^{2}\right) \sum_{l}(2 l+1) \sin ^{2}\left(\eta_{l}^{+}-\eta_{l}^{-}\right) \\
\sigma_{\mathrm{el}}=\sigma-\sigma_{\mathrm{tr}}
\end{gathered}
$$

where $\eta_{i}^{+}$and $\eta_{i}^{-}$are phase shifts for scattering by the potentials $V^{+}(r)$ and $V^{-}(r)$, respectively.
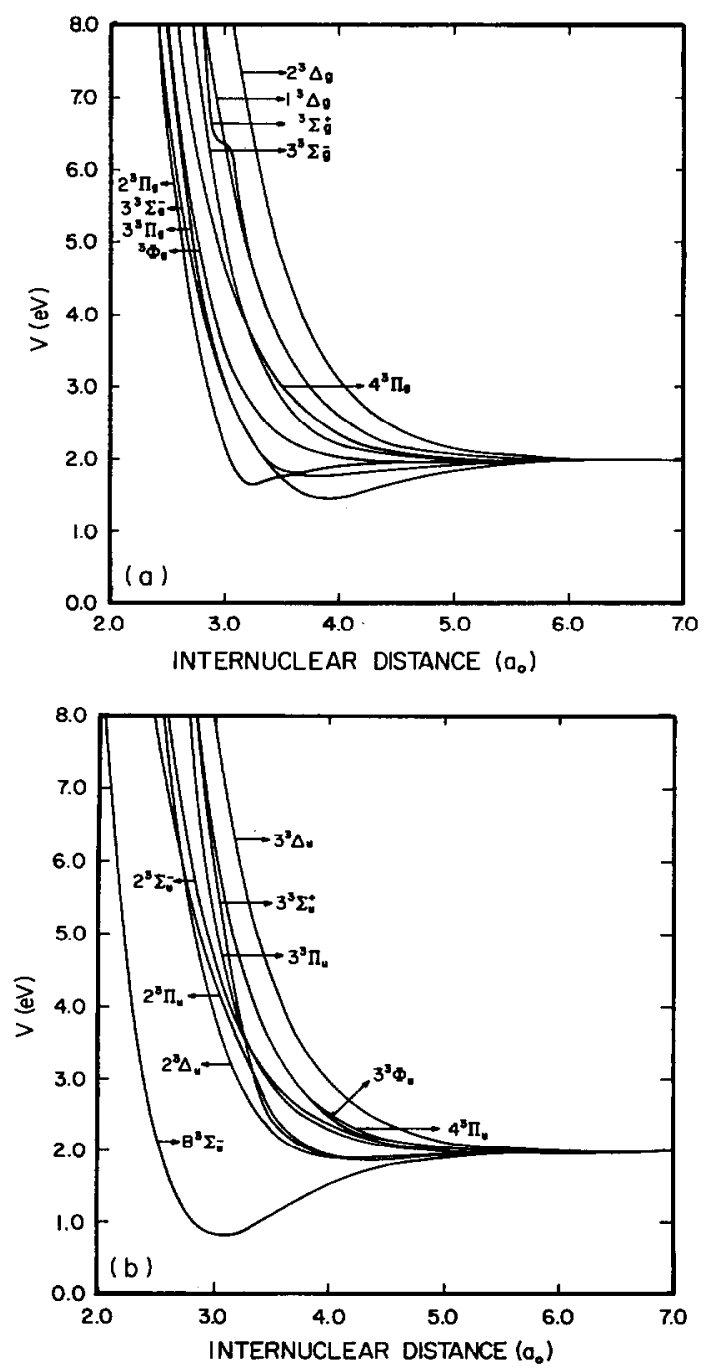

Fig. 1. THE INTERACTION POTENTIALS that SEPARATED to $\mathbf{O}\left({ }^{3} \mathrm{P}\right)-\mathbf{O}\left({ }^{1} \mathrm{D}\right)$; (a) $g$-SYMMETRY, (b) $u$-SYMMETRY.

The phase shifts were determined by solving numerically the second order differential equation

$$
\frac{\mathrm{d}^{2} F_{l}^{j}(r)}{\mathrm{d} r^{2}}+\left\{k^{2}-2 \mu V_{j}(r)-\frac{l(l+1)}{r^{2}}\right\} F_{l}^{j}(r)=0
$$

subject to the conditions $F_{l}^{j}(0)=0$ and at large $r$

$$
F_{l}^{j}(r) \sim \sin \left(k r-\frac{1}{2} l \pi+\eta_{l}^{j}\right),
$$

all quantities being measured in atomic units.

The interaction potentials $V(r)$ we adopted are the results of theoretical calculations (Saxon and Liu, 1978), modified at larger distances to be consistent 
with the long range form $C_{5} r^{-5}-C_{6} r^{-6}$. A value of 17 a.u. was adopted for $C_{6}$. The values of $C_{5}$ were then obtained by fitting to the theoretical interaction potentials at large nuclear separations. The values derived are closely equal in magnitude to those calculated by Chang (1967) for the interaction of two ground state $O\left({ }^{3} \mathrm{P}\right)$ atoms but with the opposite sign. The interaction potentials are illustrated in Fig. 1.

For the energy transfer calculations, it is convenient to introduce the angular distribution function obtained by integrating over the azimuthal angle,

$$
\sigma_{j}(\theta)=2 \pi I_{j}=2 \pi\left|f_{j}(\theta)\right|^{2}
$$

and the weighted average

$$
\sigma(\theta)=\sum_{j} g_{j} \sigma_{j}(\theta)
$$

The weighted differential cross-sections for excitation exchange, elastic and total collisions are illustrated in Figs $2 \mathrm{a}, 2 \mathrm{~b}$ and $2 \mathrm{c}$ respectively as functions of scattering angle for a relative energy, $E=\mu v^{2} / 2$, of $0.1 \mathrm{eV}$. The shadow scattering produces a characteristic sharp forward peak in the elastic collision cross-section. Because of the symmetry of the colliding species, a strong backward peak occurs in the excitation exchange cross-section. The oscillatory structure results from the existence of many bound states in the interaction potentials. Figures $3 a, 3 b$ and $3 c$ give the cross-sections for a relative energy of $1 \mathrm{eV}$. They show a similar scattering angle dependence.

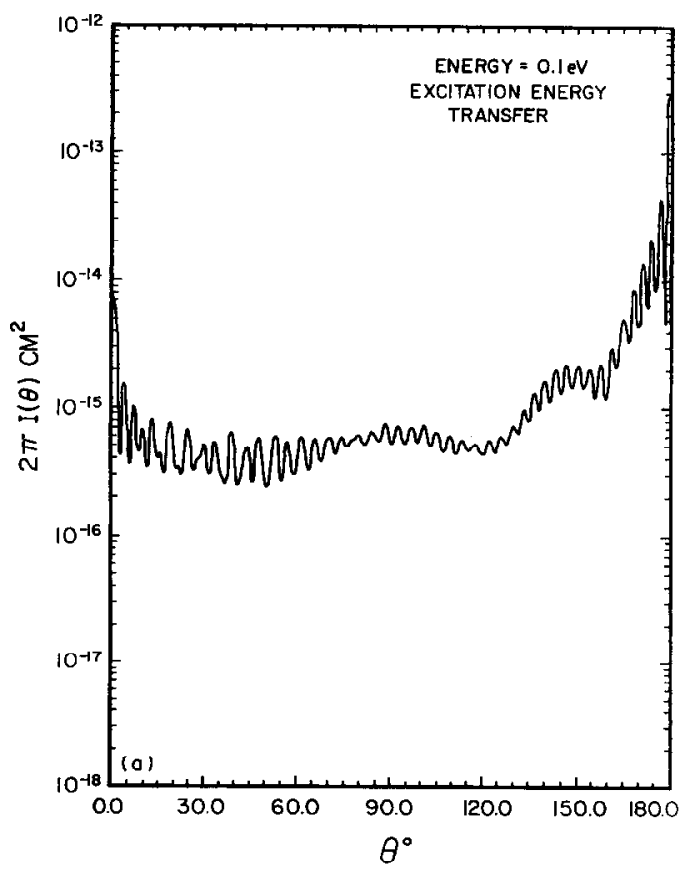

Fig. 2a.

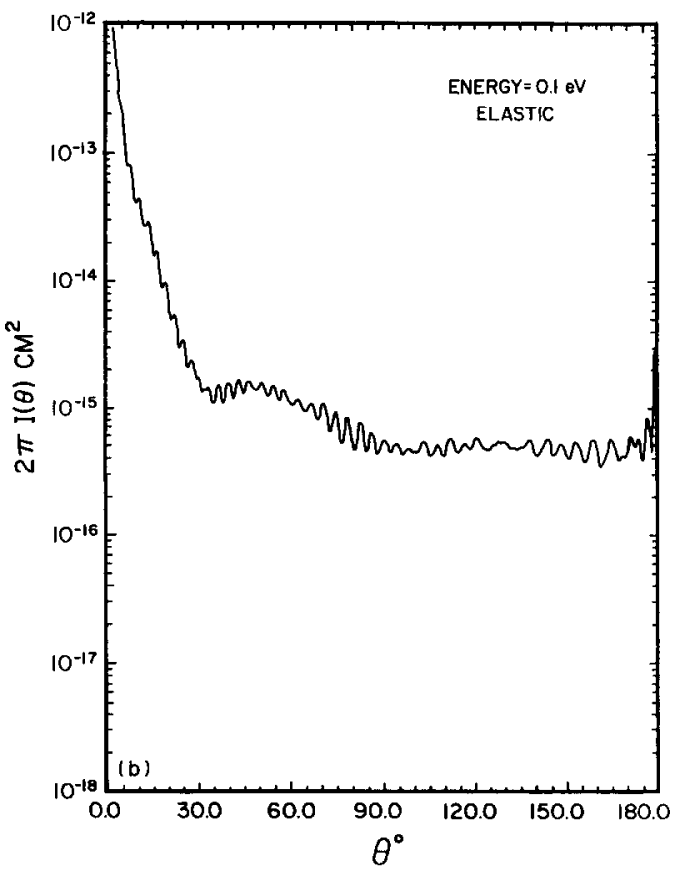

Fig. 2b.

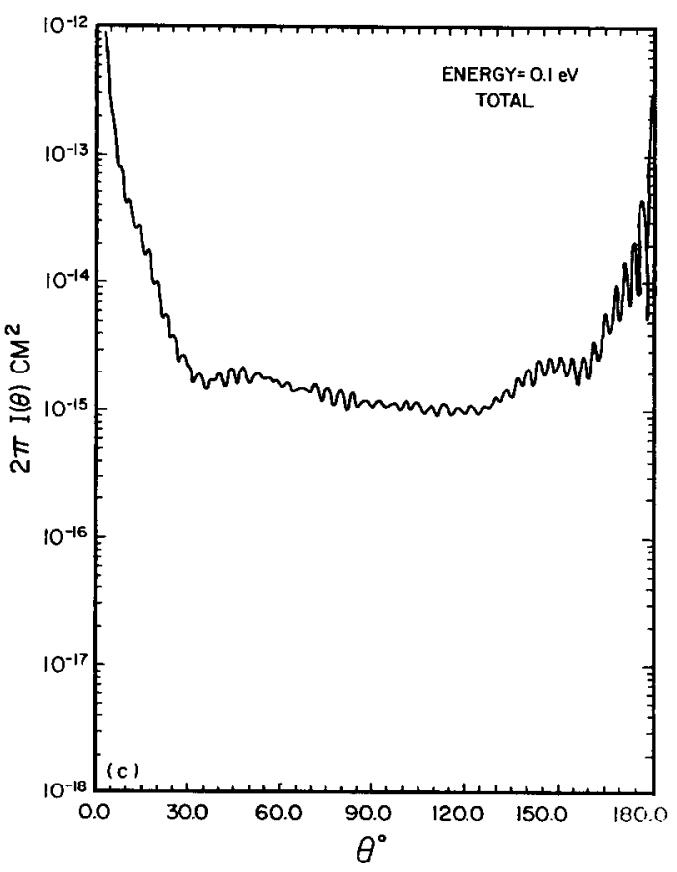

Fig. 2c.

Fig. 2. The Differential cross-Sections for (a) eXCitATION EXCHANGE, (b) ELASTIC AND (c) TOTAL COLLISIONS AS FUNCTIONS OF SCATTERING ANGLE FOR A RELATIVE ENERGY OF $0.1 \mathrm{eV}$. 


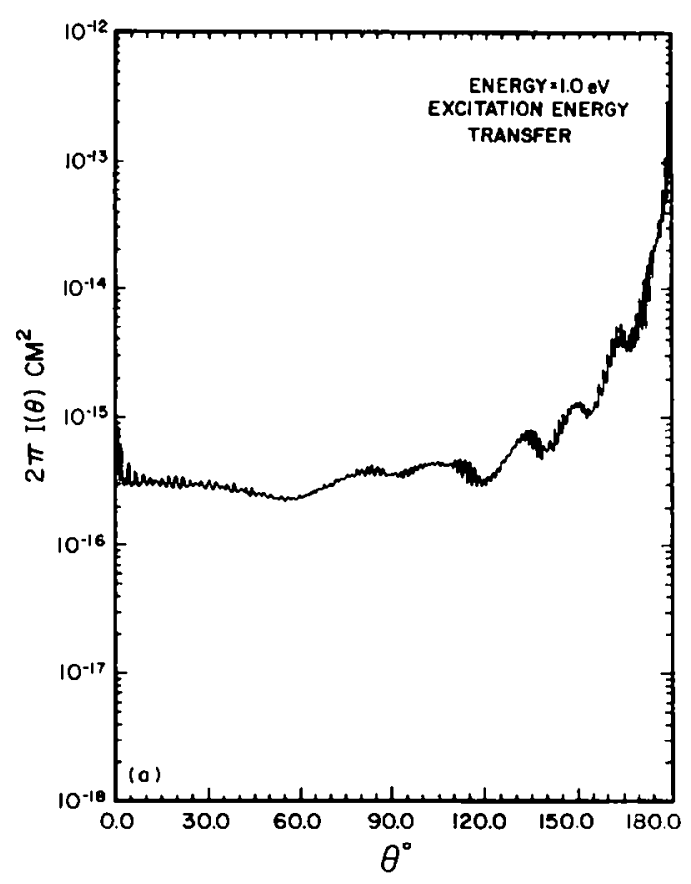

Fig. 3a.

The total scattering cross-section $\sigma$ and its contribution from excitation exchange $\sigma_{\mathrm{tr}}$ are shown in Fig. 4 as a function of energy $E$. The cross-sections vary slowly except at very low energies where the elastic collision cross-section is dominant by its sharp forward peak.

The momentum transfer or diffusion cross-section

$$
\sigma_{D}=2 \pi \int_{0}^{\pi} I(\theta)(1-\cos \theta) \sin \theta \mathrm{d} \theta
$$

is also shown in Fig. 4. The mutual diffusion coefficient of $O\left({ }^{\prime} D\right)$ and $O\left({ }^{3} P\right)$ atoms is given in terms of $\sigma_{D}$ by the formula

$$
D=\frac{3}{32} n_{0}\left(\frac{2 \pi k T}{\mu}\right)^{1 / 2} \bar{\sigma}_{D}(\bar{T})
$$

where $T$ is the temperature and

$$
\bar{\sigma}_{D}(T)=\frac{1}{2} \int_{0}^{\infty} x^{2} \sigma_{D}(x) \exp (-x) \mathrm{d} x,
$$

$x$ being $\mu v^{2} / 2 k T$. To an uncertainty of less than $2 \%$, the calculated values of $D$ may be expressed as

$$
D=\frac{2.06 \times 10^{17}}{n_{0}} T^{-0.615} \mathrm{~cm}^{2} \mathrm{~s}^{-1}
$$

where $n_{0}$ is the number density of oxygen atoms.

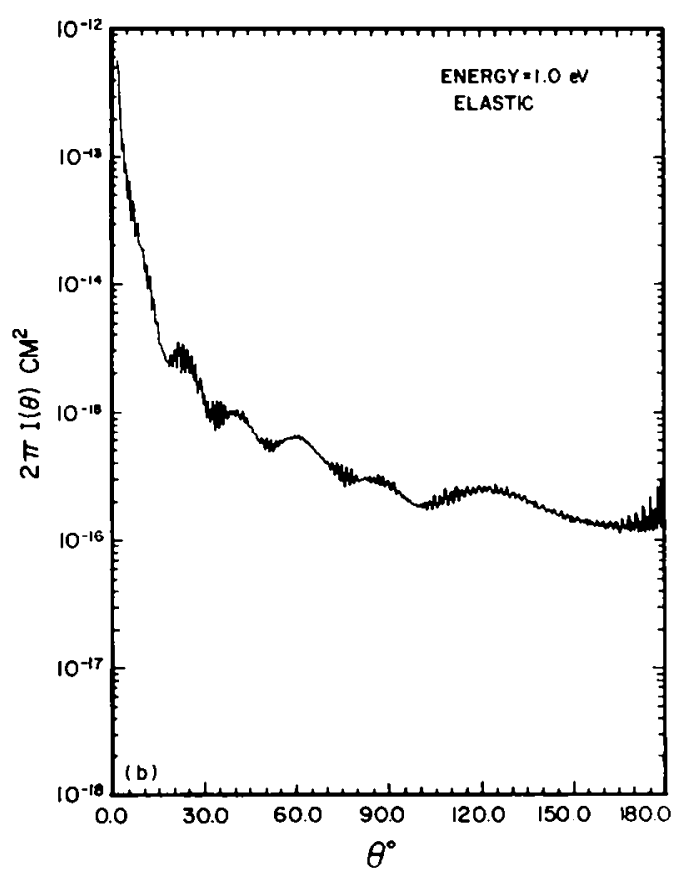

Fig. 3b.

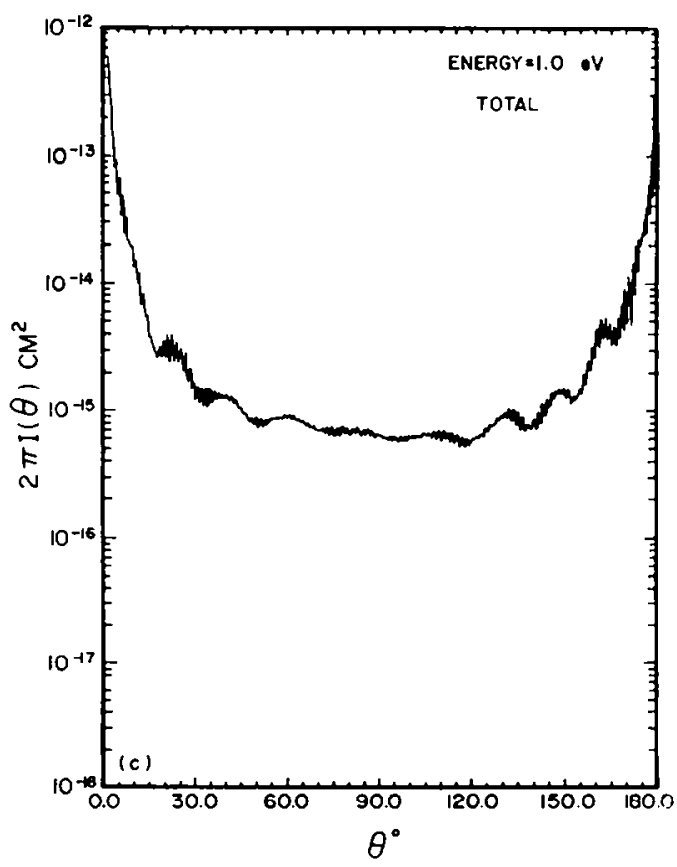

Fig. 3c.

Fig. 3. The SAME AS Fig. 2, fXCEPT FOR $E=1.0 \mathrm{eV}$. 


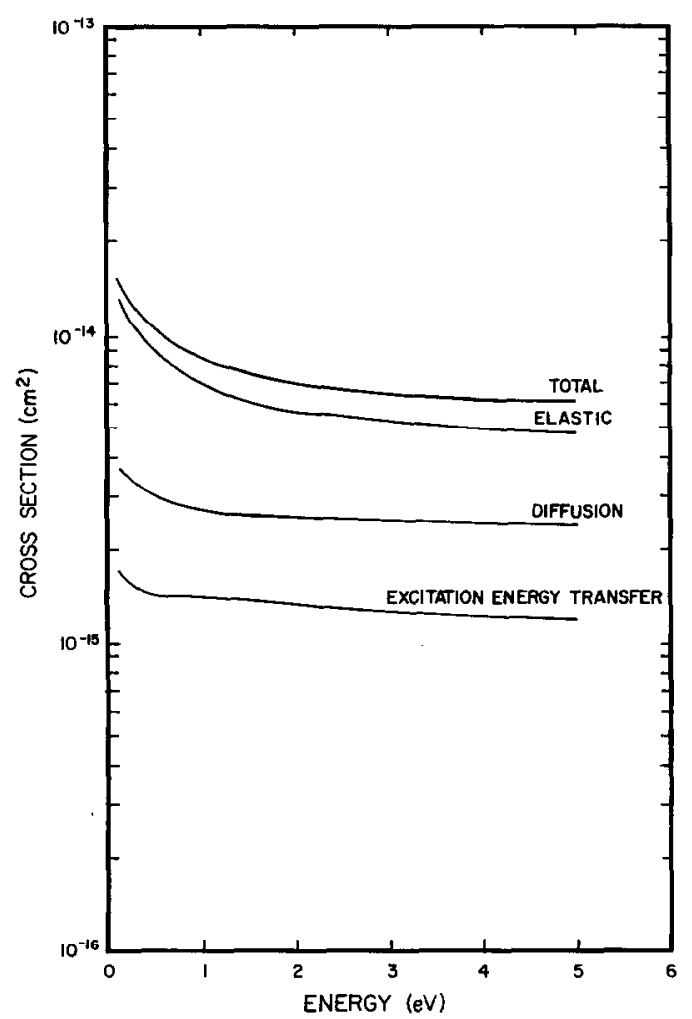

Fig. 4. Total, ELASTIC, EXCITATION EXCHANGE AND DIFFUSION CROSS-SECTIONS AS FUNCTIONS OF RELATIVE ENERGY.

\section{ENERGY TRANSFER}

The rate coefficient for the production of an $O\left({ }^{1} D\right)$ atom with energy $E_{j}$ in the collision of an $\mathrm{O}\left({ }^{1} \mathrm{D}\right)$ atom with energy $E_{i}$ and ground state $\mathrm{O}\left({ }^{3} \mathrm{P}\right)$ atoms with an energy distribution $f\left(E_{k}\right)$ may be written

$$
\begin{array}{r}
\alpha_{i j}=\bar{\sigma}_{\mathrm{el}} \int_{0}^{\infty} \int_{\chi^{-}}^{x^{+}}\left(\frac{2}{m_{0} V_{i k}}\right) f\left(E_{k}\right) \mathrm{d}(\cos \chi) \mathrm{d} E_{k} \\
+\bar{\sigma}_{\mathrm{tr}} \bar{v}_{l j} f\left(E_{j}\right)
\end{array}
$$

where $\bar{\sigma}_{t r}$ and $\bar{\sigma}_{\mathrm{el}}$ are the effective hard sphere elastic and excitation collision cross-sections respectively, defined by Yee and Dalgarno (1985), $m_{0}$ is the mass of the oxygen atom, $V_{i k}$ is the velocity of the center of mass given by

$$
V_{i k}=\left\{\frac{2\left(E_{i}+E_{k}\right)}{m_{0}}+\frac{4\left(E_{i} E_{k}\right)^{1 / 2}}{m_{0}} \cos \chi\right\}^{1 / 2},
$$

$\chi^{+}$and $\chi^{-}$are the maximum and minimum angles of $\chi$ consistent with an energy transfer $E_{i}$ to $E_{j}$, and $\bar{v}_{i j}$ is the average relative velocity defined as

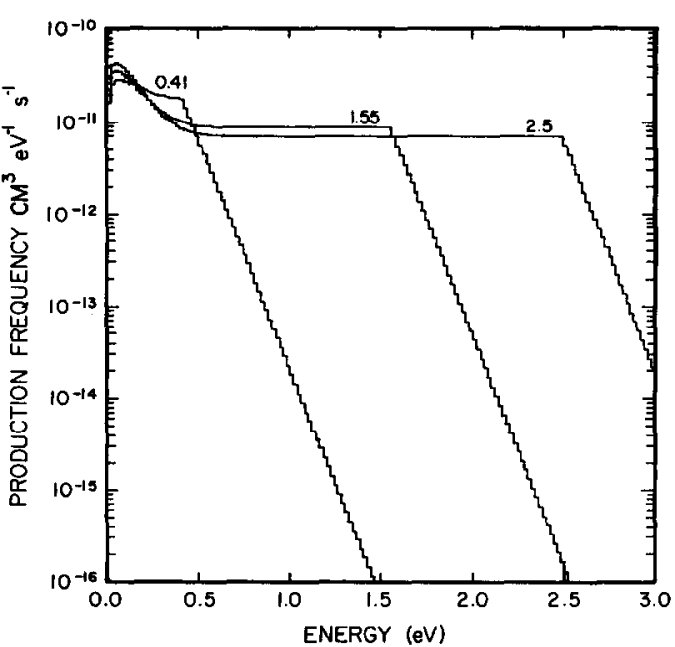

Fig. 5. The PRODUCtion FREQUENCY WITH WHICH O $\left({ }^{1} \mathrm{D}\right)$ ATOMS WITH ENERGY (a) $0.41 \mathrm{eV}$. (b) $1.55 \mathrm{eV}$ AND (c) $2.5 \mathrm{eV}$ COLLIDE WITH GROUND STATE OXYGEN ATOMS AT A TEMPERATURE OF $1000 \mathrm{~K}$ AND EMERGE WITH AN ENERGY $E_{f} \mathrm{eV}$.

$$
\bar{v}_{i j}=\frac{1}{2} \int_{-1}^{+1} V_{i j} \mathrm{~d}(\cos \chi)
$$

In defining $\bar{\sigma}_{\mathrm{el}}$ and $\bar{\sigma}_{\mathrm{tr}}$ we have omitted the small angle scattering because it does not contribute much to the thermalization process and we have considered all collisions with scattering angle greater than $160^{\circ}$ to be excitation exchange collisions (Yee and Dalgarno, 1985). For a relative energy of $1.0 \mathrm{eV}, \bar{\sigma}_{\mathrm{el}}$ and $\bar{\sigma}_{\mathrm{tr}}$ are found to be $1.55 \times 10^{-15}$ and $6.25 \times 10^{-16} \mathrm{~cm}^{2}$, respectively.

For a thermal gas at a temperature $T$,

$$
f\left(E_{k}\right)=(\pi k T)^{-3 / 2} E_{k}^{-1 / 2} \exp \left(-E_{k} / k T\right) .
$$

Figure 5 shows the production frequencies $\alpha_{i j}$ at which $O\left({ }^{1} \mathrm{D}\right)$ atoms with initial energies $E_{i}$ of $0.41,1.55$ and $2.5 \mathrm{eV}$ (one half of the excess energy from each production channel of the dissociative recombination source) are transformed to $E_{f}$ as a function of $E_{f}$ by collisions with ground state $\mathrm{O}\left({ }^{3} \mathrm{P}\right)$ atoms at a temperature of $1000 \mathrm{~K}$. In calculating $\alpha_{i j}$, we ignored the weak dependence of the cross-sections on the collision energy.

The results are similar to those reported previously for energy transfer in collisions of $\mathrm{O}\left({ }^{1} \mathrm{~S}\right)$ atoms and $O\left({ }^{3} \mathbf{P}\right)$ atoms (Yee and Dalgarno, 1985). They are important elements in the interpretation of the measured emission profiles of the red line (Schmitt et al., 1982).

Acknowledgements-This research was supported in part by 
NASA grants NAG-5-448 and NAGW-496 and by the Aeronomy Program of the National Science Foundation under Grant ATM-84-07314.

\section{REFERENCES}

Biondi, M. A. and Feibelman, W. A. (1968) Twilight and nightglow spectral line shapes of oxygen $\lambda 6300$ and $\lambda 5577$ radiation. Planet. Space Sci. 16, 431.

Biondi, M. A. and Meriwether, J. W., Jr. (1985) Measured response of the equatorial thermospheric temperature to geomagnetic activity and solar flux changes. Geophys. Res. Lett. $12,267$.

Chang (1967) Moderately long-range interaction forces. Rev. mod. Phys. 39, 911.

Froese-Fischer, C. and Saha, H. P. (1983) Multiconfiguration Hartree-Fock results with Breit-Pauli corrections for forbidden transitions in the $2 \mathrm{p}^{4}$ configuration. Phys. Rev. 28A, 3169.

Garstang, R. H. (1951) Energy levels and transition probabilities in $\mathrm{p}^{2}$ and $\mathrm{p}^{4}$ configurations. Mon. Not. R. astr. Soc. 111, 115.

Hernandez, G. (1980) Measurement of thermospheric temperaturcs and winds by remote Fabry-Perot spectrometry. Opt. Eng. 19, 518.
Ignatyev, V. M., Yugov, V. A. and Atlesov, K. V. (1984) Aurorae Airglow. Acad. Sci. U.S.S.R. 31, 134.

Mott, N. F. and Massey, H. S. W. (1965) The Theory of Atomic Collisions (3rd ed.). Oxford University Press, Oxford.

Saxon, R. P. and Liu, B. (1978) Ab initio configuration interaction study of the valence states of $\mathrm{O}_{2}$.J. chem. Phys. 67, 5432.

Schmitt, G. A., Abreu, V. J. and Hays, P. B. (1981) Nonthermal $O\left({ }^{1} D\right)$ produced by dissociative recombination of $\mathrm{O}_{2}^{+}$: a theoretical model and observational results. Planet. Space Sci. 29, 1095.

Schmitt, G. A., Abreu, V. J. and Hays, P. B. (1982) Line shape of the non-thermal $6300 \AA \mathrm{O}$ ('D) emission. Planet. Space Sci. 30, 457.

Thuillier, G., Falin, J. L. and Wachtel, C. (1977) Experimental global model of the exospheric temperature based on measurements from the Fabry-Perot interferometer on board the OGO-6 satellite discussion of the data and properties of the model. J. atmos. terr. Phys. 39, 399.

Yee, J. H. and Dalgarno, A. (1985) Energy transfer of $\mathrm{O}\left({ }^{1} \mathrm{~S}\right)$ atoms in collisions with $\mathrm{O}\left({ }^{3} \mathrm{P}\right)$ atoms. Planet. Space Sci. 33, 825 . 